OAl-PMH: http://www.indteca.com/ojs/index.php/Revista Scientific/oai

Artículo Original / Original Article

\title{
Genograma y ecomapa para identificar redes de apoyo en casos de violencia de género
}

\author{
Autoras: Karina Alexandra Garzón Jaramillo \\ Universidad Internacional SEK, UISEK \\ kagarzon.mpp@uisek.edu.ec \\ Quito, Ecuador \\ https://orcid.org/0000-0003-2976-4381
}

Elka Jacqueline Vargas Estupiñan Universidad Internacional SEK, UISEK elka.vargas@uisek.edu.ec Quito, Ecuador https://orcid.org/0000-0002-6390-3253

\section{Resumen}

Desde el año 2007 en Ecuador es declarada la violencia de género como prioridad nacional. Uno de los aspectos importantes al considerar la violencia de género son las redes de apoyo. Dos herramientas gráficas del enfoque sistémico para detectar redes de apoyo es el genograma que al dibujar un árbol familiar se puede reconocer los potenciales apoyos dentro de la familia. Y, el ecomapa, que consiste en dibujar círculos alrededor de la familia que representan a los sistemas externos que tiene la familia. El objetivo que guía esta investigación es considerar el uso del genograma y el ecomapa para la identificación de redes de apoyo en casos de violencia de género. La investigación es de tipo descriptivo, transversal, inductivo, cualitativo. Se llevó a cabo una entrevista a 5 usuarios de la Unidad de Víctimas de la Defensoría Pública y a 10 funcionarios que laboran en la misma Unidad. Se concluye que las mujeres víctimas de violencia de género se benefician del genograma y ecomapa, al reconocer gráficamente su historia de vida y sus potenciales apoyos. Y los profesionales de primera acogida indistintamente de su profesión podrían capacitarse y usar estas herramientas para una atención integral a esta población.

Palabras clave: violencia; redes de apoyo; familia; sistemas externos. Código de clasificación internacional: 6112.01 - Discriminación.

Cómo citar este artículo:

Garzón, K., \& Vargas, E. (2021). Genograma y ecomapa para identificar redes de apoyo en casos de violencia de género. Revista Scientific, 6(22), 117-137, e-ISSN: 2542-2987. Recuperado de: https://doi.org/10.29394/Scientific.issn.2542-2987.2021.6.22.6.117-137

Fecha de Recepción: 15-01-2021
Fecha de Aceptación: 04-06-2021
Fecha de Publicación: 05-11-2021 


\title{
Genogram and eco-map to identify support networks in cases of gender violence
}

\begin{abstract}
Since 2007, in Ecuador gender violence has been declared a national priority. One of the important aspects when considering gender-based violence is support networks. Two graphic tools of the systemic approach to detect support networks is the genogram that when drawing a family tree it is possible to recognize the potential supports within the family. And, the ecomap, which consists of drawing circles around the family that represent the external systems that the family has. The objective that guides this research is to consider the use of the genogram and the eco-map for the identification of support networks in cases of gender violence. The research is descriptive, transversal, inductive, qualitative. An interview was carried out with 5 users of the Victims Unit of the Public Defender's Office and 10 officials who work in the same Unit. It is concluded that women victims of gender violence benefit from the genogram and ecomap, by graphically recognizing their life history and their potential supports. And first reception professionals, regardless of their profession, could be trained and use these tools for comprehensive care for this population.
\end{abstract}

Keywords: violence; support networks; family; external systems. International classification code: 6112.01 - Discrimination.

How to cite this article:

Garzón, K., \& Vargas, E. (2021). Genogram and eco-map to identify support networks in cases of gender violence. Revista Scientific, 6(22), 117-137, e-ISSN: 2542-2987. Recovered from: https://doi.org/10.29394/Scientific.issn.2542-2987.2021.6.22.6.117-137

Date Received: 15-01-2021
Date Acceptance: 04-06-2021
Date Publication: 05-11-2021 


\section{Introducción}

Los datos de violencia de género a nivel mundial dan cuenta de una realidad que alarma sobremanera. Lamentablemente, se relativiza a través de las palabras y se mantiene a pesar de la guerra declarada en contra de este fenómeno humano llamado violencia.

Para la Organización de las Naciones Unidas (ONU, 1979): el Estado Ecuatoriano acogió la obligación internacional de la lucha contra la violencia de género con la adopción del Convenio para la Eliminación de todas las formas de Discriminación contra la Mujer. Desde el año 2007 se considera como prioridad nacional la erradicación de la violencia de género, desde un marco de defensa de los derechos humanos y que conlleve a un tratamiento integral de la problemática. El Instituto Nacional de Estadística y Censos (INEC, 2019): de acuerdo con los resultados de la última Encuesta Nacional sobre Relaciones Familia y Violencia de Género contra las Mujeres, indica que el 43 de cada 100 mujeres en Ecuador sufrieron violencia de pareja a lo largo de su vida. Así mismo, 18 de cada 100 mujeres en el país experimentaron algún tipo de violencia de parte de su pareja en los últimos 12 meses. Entre el $82 \%$ y el $96 \%$ de las víctimas de violencia física, entre el $89 \%$ y el $97 \%$ de las víctimas de violencia psicológica y el $81 \%$ y el $95 \%$ de las víctimas de violencia sexual y patrimonial NO colocaron una denuncia a sus agresores.

Estas cifras dan cuenta de la naturalización de la violencia en nuestro contexto, la falta de conciencia de la problemática y el escaso conocimiento de los derechos de las mujeres. Apenas un mínimo porcentaje busca un respaldo legal y muy pocas personas se permiten una atención médica y/o psicológica.

La búsqueda y aplicación de estrategias parta dar una respuesta a esta problemática ha sido amplia pero aún insuficiente, dada su permanencia e incremento. Definitivamente, de acuerdo con Estrada, Herrero y Rodríguez (2012): las mujeres víctimas de violencia necesitan espacios terapéuticos 
donde puedan reconstruir sus historias encontrando soluciones a la permanencia en el ciclo de violencia. Entre otros factores, una de estas soluciones se encuentra en el fortalecimiento de factores protectores, como la creación de redes de apoyo.

Las redes de apoyo aluden al concepto de apoyo social analizado por Lin, Dean y Ensel (1986): que lo definen como provisiones de tipo instrumental y/o expresivo dadas por la comunidad, redes sociales y personas cercanas que generan confianza.

A partir de esta concepción se han desarrollado investigaciones en las que el apoyo social tiene un efecto sobre la salud mental y el bienestar ante circunstancias vitales difíciles e inclusive para superar situaciones de violencia. La idea de contar con la cercanía o pertenencia a una red de apoyo es totalmente contraria a la unicidad y aislamiento. Las redes de apoyo son acordes a la naturaleza gregaria del ser humano; que se manifiesta a través de la tendencia a vivir en comunidad en-con-por-para otros.

En este sentido, cuando una persona vive situaciones traumáticas en su historia, como violencia de género, se requieren redes de apoyo para disminuir o superar estas situaciones generadoras de estrés, precisamente este factor estresante influye en que la persona tenga una visión reduccionista de los recursos que dispone, por lo que tiende a minimizar el apoyo social. De acuerdo con Fernández (2005): el potencial de la red de apoyo va más allá del entramado de recursos, se centra en la percepción que la persona tiene de su entorno ya que existen personas que reciben apoyo, pero no lo perciben, si no hay la percepción de un apoyo disponible, éste no podrá ser utilizado.

Sin embargo, dentro de las atenciones psicológicas y de apoyo psicosocial no existe una definición clara de las herramientas que se podrían utilizar para identificar adecuadamente estas redes, puesto que no todas las personas que conforman una red social pueden ser potencialmente apoyos para una mujer que vive violencia de género. 


\subsection{El sistema familiar $y$ otros sistemas}

El ser humano se constituye con relación a otros y a través de todos los significantes que le son depositados. Esos otros en primera instancia son los cuidadores primarios y personas del entorno inmediato que comprenden a la familia y que luego va ampliándose e interactuando con los miembros y/o instituciones de otros sistemas externos en donde se desarrolla y se sostiene.

El Enfoque Sistémico Familiar, conforme a Bowen (1998): permite una comprensión de la familia desde sus dinámicas y relaciones en donde cada miembro influye y contribuye en la formación de todos los integrantes. Se vincula de igual forma el Modelo Ecológico de Bronfrenbrenner (1987a): el cual considera que la conducta del ser humano se ve afectada e influenciada por los ambientes naturales, es decir, que está en continuo proceso de acomodación por sus interconexiones con sus entornos inmediatos que también son cambiantes.

De esta manera, Bronfrenbrenner (1987b): concibe a los contextos de manera concéntrica, donde los sistemas y subsistemas conforman el ecosistema que posibilitan el desarrollo del ser humano, parte desde el individuo en relación con sus sistemas desde lo familiar que representa lo más cercano hasta el sistema mayor que es la sociedad. Estos son cuatro: microsistema, mesosistema, exosistema y macrosistema:

1. Microsistema: Es el nivel más cercano donde se producen relaciones interpersonales directas.

2. Mesosistema: Es un sistema de microsistemas que se interrelacionan $y$, donde la persona activamente participa. Pueden ser estructuras formales o informales como la familia ampliada, vecindad, amistades, escuela, trabajo, religión, ámbito sanitario con las que se mantienen relaciones durante el desarrollo.

3. Exosistema: Comprende uno o más entornos donde la persona no tiene una participación activa sin embargo recibe su influencia. 
4. Macrosistema: Representa a la cultura, con sus valores e ideología.

El Cronosistema lo plantea Bronfrenbrenner (1994c): en el que engloba los demás subsistemas en una instancia mayor, representada por la línea del tiempo en el ambiente en el que vive y que generan cambios en su desarrollo.

Tomando en cuenta los sistemas y subsistemas del Modelo Ecológico, para Navarro y Rodríguez (2010): se rescata como herramientas útiles del Enfoque Sistémico al genograma y ecomapa, las dos son técnicas gráficas que pueden ser adaptadas y usadas en proporción a las necesidades y perspectivas por diversos profesionales, no sólo del área de salud, sino también por antropólogos, historiadores, médicos familiares, genetistas, trabajadores sociales, enfermeras, psicólogos, etc. Interpretando a McGoldrick y Gerson (2000a): el genograma es un formato para dibujar un árbol familiar que registra a través de símbolos a los miembros de la familia y sus relaciones.

A partir del genograma se grafica el ecomapa, al dibujar círculos alrededor que representan a los sistemas externos con los que tienen relación directa con la familia, como: familia extensa, amistades, vecindad, salud, educación, trabajo, deporte, religión, cultura y recreación. Además, se trazan líneas entre estos sistemas que unen a la familia o algunos miembros que dan cuenta del tipo de relaciones.

Al contar con un gráfico de redes de apoyo la mujer víctima de violencia de género se permite visualizar y diferenciar de una manera más clara si cuenta o no con personas que pueden darle apoyo material y, principalmente emocional.

En este contexto aparece el cuestionamiento: ¿El genograma y ecomapa para identificar redes de apoyo puede ser usado por profesionales de primera acogida en casos de violencia de género? 


\subsection{Haciendo caminos: redes de apoyo}

Esta investigación tiene como objetivo rescatar la importancia de enfocar el proceso psicoterapéutico o de apoyo psicosocial en la búsqueda de soluciones ante la violencia de género a partir de la visualización de las redes de apoyo con técnicas gráficas como el genograma y ecomapa que permiten organizar y definir con mayor claridad la estructura y dinámica familiar y así, detectar las personas-apoyo dentro del sistema familiar $y$ complementariamente las personas o instituciones que son apoyos fuera del hogar.

Comprender al ser humano y sus interacciones desde el modelo ecológico permite entender de mejor manera la violencia de género e identificar los factores de protección para enfrentar la problemática. Se trata además de una recomendación que la Organización Mundial de la Salud (OMS, 2002): viene realizando desde años atrás, puesto que considera a la violencia de naturaleza polifacética y, por tanto, requiere ser abordada desde el enfoque ecológico, el mismo que empezó a utilizarse en la década de 1970 al estudiar el maltrato a niños, modelo que incluso se aplicó a ulteriores estudios.

En este orden de ideas, Sluzki (1996): considera que la red social es un conjunto de relaciones interpersonales representativas (vínculos afectivos) para el sujeto. Las funciones del apoyo social se sitúan en el ámbito instrumental, informacional y emocional. El instrumental se refiere a que las relaciones sociales son un medio para que la persona logre sus objetivos, tales como conseguir empleo o contar con apoyo en el cuidado de los niños/as.

El ámbito informacional se basa en la comunicación verbal representada en palabras o consejos dados por la red. Por último, para Gracia (1997): la dimensión emocional da cuenta de los vínculos interpersonales en los que hay un clima de comprensión y simpatía que permite expresar sentimientos y en donde se busca la aceptación por los demás miembros de 
la red.

Es importante tomar en cuenta que no todas las redes sociales son útiles para cubrir las necesidades materiales y emocionales de las personas, es decir no todas las redes sociales se configuran como fuente de ayuda, las redes de apoyo significativas están orientadas a generar un mayor bienestar a aquel integrante de la red que requiere del apoyo.

Existen personas e instituciones que toleran y perpetúan la violencia a través de roles de género y estereotipos, a pesar de ser parte de la red social de una mujer que vive violencia no necesariamente es un apoyo. La red social, siguiendo a Rodrigues y Cantera (2017): puede reeditar la violencia al minimizar su sufrimiento y culpabilizarla, en lugar de escucharla y validar su experiencia, es decir la red social asume un rol negativo y disfuncional que se denomina "victimización secundaria" y que genera heridas psicológicas.

De esta manera, en un proceso psicológico y de apoyo psicosocial es importante trabajar la multiplicidad de variables y aristas que tiene la violencia, entre ellas las redes de apoyo. Una estrategia eficaz para la intervención es valerse de herramientas sistémicas como el genograma y el ecomapa, que permiten visualizar las redes de apoyo con las que cuenta una mujer víctima de violencia de género.

\subsection{Apostando a una mejor intervención}

El Enfoque Sistémico, como indican Minuchin y Fishman (2004): aborda al ser humano desde el aspecto relacional y comunicacional, por eso es indefinible sino está ligado al análisis del sistema familiar, en donde los miembros tienen una causalidad circular y es considerado como una totalidad en un sistema caracterizado por límites, roles y funciones. Mencionando a Pérez (2016): el protagonismo de un síntoma o enfermedad en uno de los miembros afecta a todos los demás del sistema.

En ese marco Satir (1986): plantea que todos los miembros de la familia 
son parte del problema y la mejor manera de abordarlo es a través del análisis de las relaciones y comunicaciones familiares. Una de las técnicas ampliamente utilizada es el genograma creado por Bowen (1978): y difundido en el contexto clínico por McGoldrick y Gerson (2000b): que gráficamente representa la estructura y dinámica familiar y permite, para este caso, a la persona víctima de violencia ubicarse en la trama de su vida y mirarse como parte de una red; según La Moneda (2010): de esa manera se autoriza así mima para hacerse fuerte.

Otra herramienta complementaria para redes de apoyo es el ecomapa o mapa ecológico de la familia creada por Hartman (1979): en el área de trabajo social, parte del genograma y colocando círculos alrededor permite reconocer personas o instituciones, y sus conexiones con influencia positiva o negativa a la familia en general o para algunos miembros específicamente. El principal efecto de esta herramienta es el impacto visual que genera en la persona, por los recursos con los que cuenta y aquellos que necesita modificar.

En el proceso psicológico o de apoyo psicosocial estas dos técnicas se pueden conjugar con la intención de buscar y reforzar redes de apoyo en la mujer víctima de violencia de género disminuyendo el tiempo de intervención y aumentando la calidad de los resultados obtenidos.

Esta investigación desea resaltar la factibilidad de que dentro de un proceso psicológico o de apoyo psicosocial los profesionales de primera acogida podrían usar el genograma complementariamente con el ecomapa en favor de la identificación temprana de apoyos dentro y fuera de la familia y así logren visualizar de manera ordenada y gráfica los recursos de soporte, protección, amparo o defensa y puedan distinguir de aquellas redes sociales que influyen negativamente en su persona y su dinámica familiar, para considerar su retiro o la posibilidad de modificarlas o cambiarlas.

Es importante resaltar que el objetivo de esta investigación es considerar el uso del genograma y el ecomapa para la identificación de redes 
de apoyo en casos de violencia de género.

\section{Metodología (Materiales y métodos)}

La investigación es de tipo descriptivo, transversal, inductivo, tipo cualitativo. La interpretación de los datos de entrevistas, se la realizó a través de historias de vida y el análisis de datos de la encuesta, con estadística descriptiva. La población estuvo conformada de 5 mujeres víctimas de violencia de género de la Unidad de Víctimas de la Defensoría Pública y 10 funcionarios de acogida de la misma unidad.

Esta investigación estuvo dentro del Proyecto Redes de apoyo en mujeres víctimas de violencia, desarrollado por la Universidad Internacional SEK (UISEK), la Universidad de Guadalajara (UdeG) y la Defensoría Pública del Ecuador, el cual contó con la aprobación del Comité Científico de dichas instituciones, mencionando a Laso (2019a): así se generó un cuestionario para ser llenado, se utilizó el Cuestionario de apoyo en Pareja y Estereotipos.

Se realizó una entrevista única de 90 minutos con cada mujer víctima de violencia de género, en primera instancia se realizó la revisión y firma del consentimiento informado, la aplicación de los 73 ítems del cuestionario y posteriormente se aplicó las técnicas del genograma y ecomapa para identificar las posibles redes de apoyo y diferenciarlas de las redes sociales que no son beneficiosas. La recolección de datos estuvo orientada a proveer de un mayor entendimiento de los significados y experiencias de las participantes.

Por otra parte, previo consentimiento informado se aplicó a 10 funcionarios de primera acogida de la Unidad de Víctimas de la Defensoría Pública, una encuesta semiestructurada que fue validada por dos expertos en la temática, el cual constó de 10 preguntas cerradas respecto al uso y aplicación de herramientas para la identificación de redes de apoyo en caso de violencia de género, y cuyas respuestas se registraron en escala Likert: 
alto, medio y bajo. Esta información se sometió a análisis estadístico descriptivo. Es importante mencionar que no se dejó de lado las apreciaciones y comentarios que los funcionarios realizaron al finalizar la encuesta.

\section{Resultados (análisis e interpretación de los resultados)}

La nube de palabras de la figura 1, condensa los significantes que estuvieron presentes en las historias de vida de las cinco mujeres que fueron entrevistadas y con las cuales se elaboró genogramas y ecomapas con el objetivo de identificar redes de apoyo dentro y fuera de la familia. Además, se añade los porcentajes de los datos procesados de la Encuesta Cuestionario de apoyo en pareja y estereotipos que fue realizada en Quito y Ambato a 75 mujeres con edades comprendidas entre 14-69 años.

Figura 1. Significantes entorno a redes de apoyo.

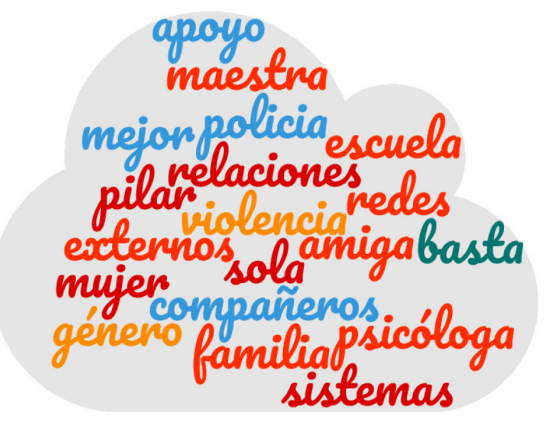

Fuente: Las Autoras (2020).

Durante el desarrollo del genograma se pudo identificar relaciones distantes y hasta conflictivas con sus progenitores al igual que con sus hermanos. Así, lo relatan las participantes 1, 3 y 4, respectivamente: Mi mamá siempre decía, aunque pegue o mate marido es. Mi padre, siempre me rechazó, e incluso llegó a decir que no era su hija. Me vinieron a pegar, mi mamá casi me mató... me pegó en la cabeza, me cocieron. Decidí ya no volver a Ambato... Con nadie nos llevamos, chocamos en el camino, me tuercen la 
boca, me hacen a un lado desde hace seis años, cuando me separé, empecé a vivir en Quito y Tanto mi mami como mi papi me han fallado, en algún momento me hicieron daño, siempre salían discutiendo, nunca se llevaron bien, me ponían en el medio a mí.

Con respecto a los hijos, cuatro participantes tienen hijos y tres mencionaron que sienten respaldo de parte de ellos, así lo relataron las participantes 1,3 y 5 respectivamente: Él sufrió bastante, traía folletos de alcohol y drogas de la escuela, y me decía: Ya basta mamá si tú también vales, Desde que mi mamá falleció me empecé a llevar con mis hijos, porque les ponía en contra mío. Ahora estamos en contacto y Siempre han estado conmigo.

En los cuatro casos, las parejas actuales no son maltratadoras y mantienen relaciones satisfactorias, según mencionaron las participantes 1, 2, 3 y 5: Él es una persona muy seria, me decía: Lo que tú estás haciendo no está bien, Mi pareja siempre ha estado ahí, apoyando, haciéndome ver las cosas. Con mi pareja actual, nos llevamos bien, me cuida y Mi enamorado me trata muy diferente que mi ex.

A continuación, en la figura 2, se expone uno de los genogramas que se realizó con una participante.

Figura 2. Genograma construido con la participante 2.

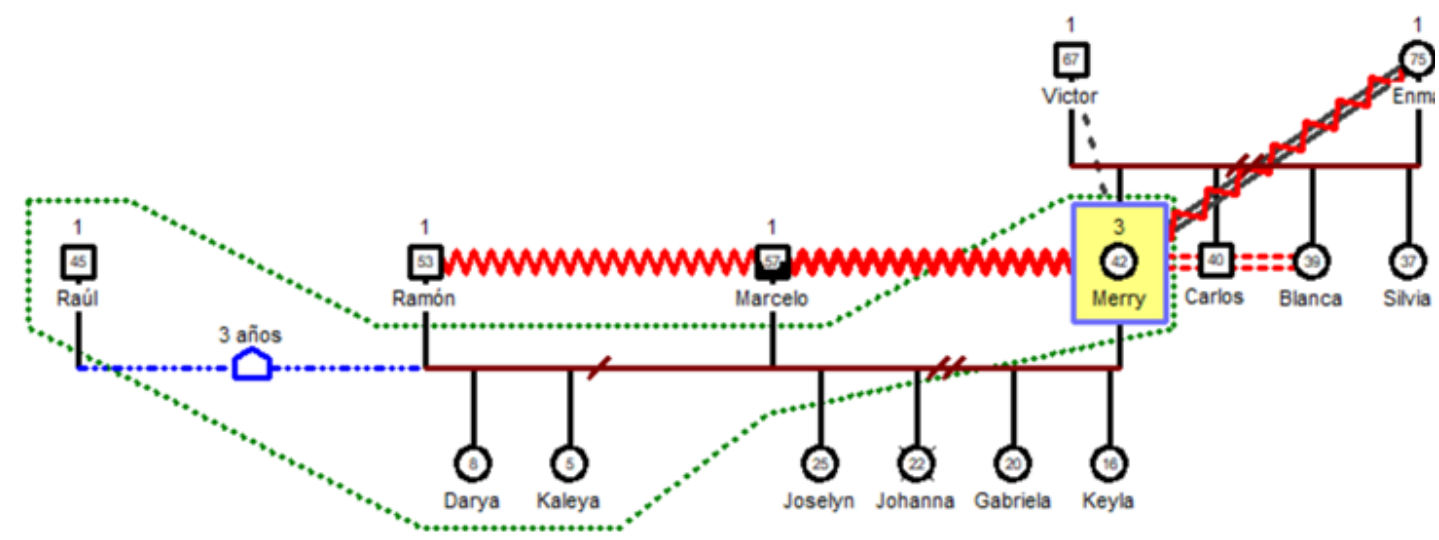

Fuente: Las Autoras (2020). 
Por otra parte, al realizar complementariamente el ecomapa, se encontró que los usuarios entrevistados rescatan a personas externas de la familia que representan un apoyo como: mejor amigo/a, participante 1, relata: Él andaba conmigo siempre, me iba a ver, me llama todos los días, cómo estoy, si amanecí bien. Siempre ha estado ahí conmigo. La vecina, participante 5: Mi vecina que es mi comadre, aunque sea con un platito de comida ha estado conmigo. La policía, así menciona la participante 2: Cuando el papá de mis hijos me golpeaba, me fui donde ellos y me dijeron: tiene q sacar una boleta de auxilio, sino le puede hasta matar. Desde hace 12 años tengo la boleta de auxilio. La psicóloga, participante 1 , relata que ahora la psicóloga me dijo que no me vaya (a la Costa, donde su madre) si me afecta, antes pasaba horas conversando con ella, me hiere y me lastima.

En la figura 3, se presenta un genograma y ecomapa realizado junto a una de las mujeres entrevistadas.

Figura 3. Genograma y ecomapa construido con la participante 1.

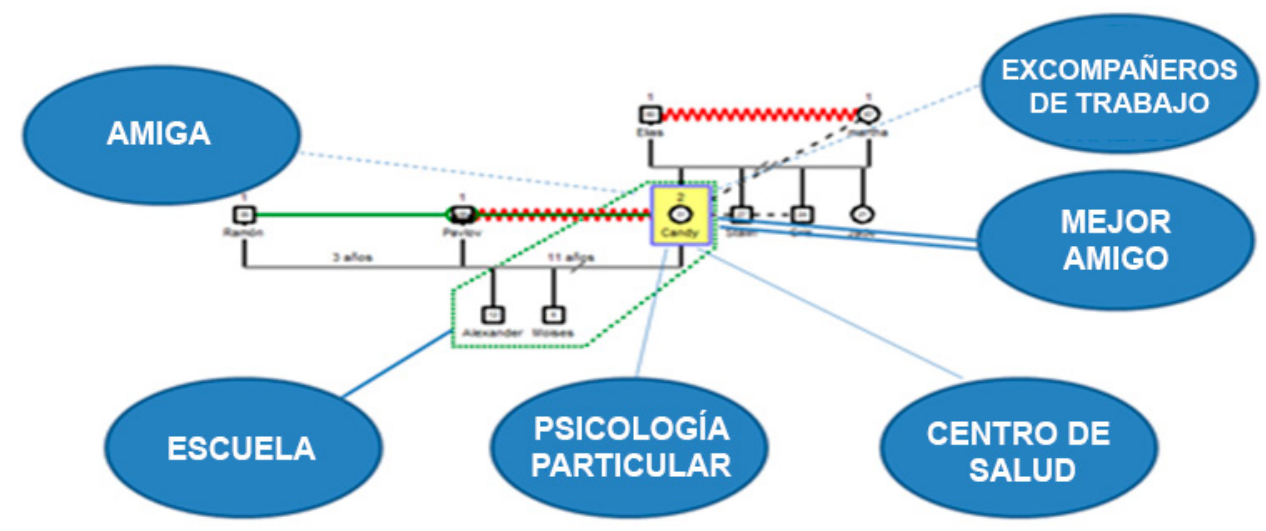

Fuente: Las Autoras (2020).

Finalmente, hubo personas e instituciones que algunos usuarios refirieron no ser apoyos, entre ellas: 
Con respecto a la familia extensa, sólo una participante mencionó a la esposa de su tío como un apoyo y el resto lo resaltó contrariamente a un apoyo. Se menciona las experiencias de las participantes 1 y 4 . Así, ....un tío me iba a pegar diciendo que me he separado, le dije que no tiene que meterse en mi vida, no sabe ni las cosas que están pasando y Siempre me fallan todos, desde mi casa (alude a padres y tíos maternos). Me deslinde de todos ellos a partir del fallecimiento de mi abuelita.

Gráfico 1. Nivel de confianza en terceras personas (Cuestionario de apoyo en pareja y estereotipo). Si estuvieras en una seria crisis personal, ¿Cuántas personas crees que tratarían de ayudarte?.

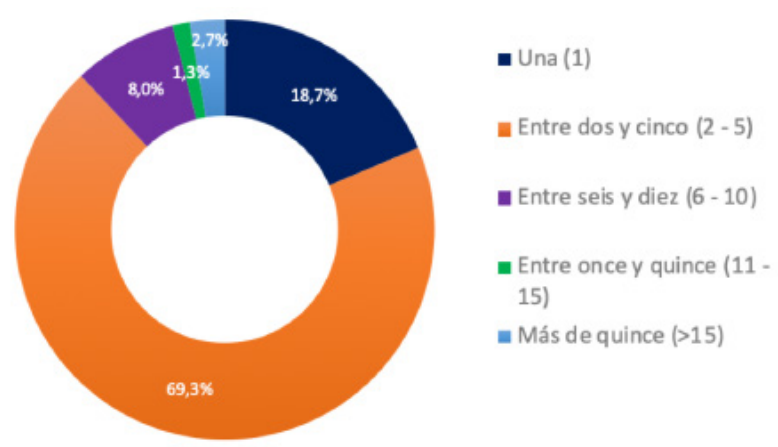

Fuente: Laso (2019). 
Finalmente, en el gráfico 1 y gráfico 2, es necesario rescatar que la percepción de redes de apoyo es reducida en estos casos de violencia de género, consideran el $18,7 \%$ que sólo una persona trataría ayudarla frente a una crisis personal y el $69,3 \%$ mencionan que serían entre 2-5 personas máximo. El 94,7\% estiman necesario cuidarse las espaldas porque casi nadie es confiable.

En cuanto a los resultados obtenidos de las entrevistas semiestructuradas hacia los 10 funcionarios de acogida de la Unidad de Víctimas de la Defensoría Pública, se encuentran:

Más de la mitad de los entrevistados respondieron que tiene un conocimiento mediano del uso del genograma como herramientas de identificación de redes de apoyo, este conocimiento no fue recibido en su formación de grado, más bien fue en formaciones de posgrado en el área de Derechos Humanos y en capacitaciones inherentes al tema de género.

Casi todos los entrevistados desconocían o conocían escasamente el término ecomapa y sus implicaciones. Por esta razón todas las puntuaciones con respecto a ecomapa son bajas, excepto el interés que los profesionales tienen en recibir capacitación de esta herramienta, la mayoría tienen un interés medio-alto en recibir un curso en el uso de genograma y ecomapa como herramienta para identificación de redes de apoyo en casos de violencia de género, como se puede apreciar en los resultados de la tabla 1.

Tabla 1. Resultados de la encuesta a funcionarios de la Unidad de Víctimas de la Defensoría Pública.

\begin{tabular}{|l|c|c|c|}
\hline \multicolumn{1}{|c|}{ CATEGORÍAS } & ALTO & MEDIO & BAJO \\
\hline Conocimiento del uso del genograma. & & X & \\
\hline Conocimiento del uso del ecomapa. & & & X \\
\hline Genograma: herramienta para disminución de casos. & X & X & \\
\hline Ecomapa: herramienta para disminución de casos & & & X \\
\hline Genograma es posible usarla en la práctica. & $\mathrm{X}$ & $\mathrm{X}$ & \\
\hline Ecomapa es posible usarla en práctica. & & $\mathrm{X}$ & $\mathrm{X}$ \\
\hline Formación profesional incluyó el genograma. & & & $\mathrm{X}$ \\
\hline
\end{tabular}




\begin{tabular}{|l|c|c|c|}
\hline Formación profesional incluyó el ecomapa. & & & $\mathrm{X}$ \\
\hline Interés en capacitarse en el uso de genograma. & $\mathrm{X}$ & $\mathrm{X}$ & \\
\hline Interés en capacitarse en el uso de ecomapa. & $\mathrm{X}$ & $\mathrm{X}$ & \\
\hline
\end{tabular}

Fuente: Las Autoras (2020).

Todo esto se presenta con la finalidad de aplicarlas en sus intervenciones profesionales, además de rescatar las redes de apoyo, algunos los consideraron como herramientas para organizar y tener mayor claridad del entorno familiar y social del defendido e incluso poder identificar testigos a favor para el caso. Dos funcionarios mencionaron: Yo si los escucho, es diferente de revictimizar, Veo práctico el uso del genograma como una ayuda gráfica para teoría del caso, seguimiento, ver testigos y en la audiencia oral en el tribunal.

\section{Conclusiones}

No todas las personas o instituciones que conforman la red social de una mujer víctima de violencia representan un apoyo. Los profesionales de primera acogida que atienden casos de violencia de género podrían verse beneficiados por el uso del genograma y ecomapa como ayuda gráfica para teoría del caso e identificación de testigos a favor del defendido.

Existe el deseo de recibir capacitación en genograma y ecomapa por parte de los funcionarios de la Defensoría Pública, en aras de un trabajo interdisciplinario, donde es erróneo considerar a las herramientas sistémicas exclusivas de escasos profesionales del área de salud, como: médicos, psicólogos o trabajadores sociales.

Las mujeres víctimas de violencia de género se benefician de estas herramientas (genograma y ecomapa) al reconocer gráficamente su historia de vida y sus potenciales apoyos dentro y fuera del hogar.

Identificar las redes de apoyo en casos de violencia de género permite conducir a la persona hacia un mayor bienestar al reconocer sus apoyos, los 
apoyos potenciales que faltan reforzar o aquellos que tienen una función negativa que generan un desgaste en todas sus dimensiones.

Se tiene en cuenta que la aplicación de estas herramientas no es la solución al gran problema de salud que representa la violencia de género, pero al darle un abordaje desde el Modelo Ecológico existe una mayor posibilidad de dar una respuesta a esta problemática, desde la trinchera que uno este. Como profesionales de primera acogida indistintamente de la profesión y orientación se direccionan esfuerzos hacia una atención integral de esta población.

\section{Referencias}

Bowen, M. (1998). De la familia al individuo: La diferenciación de sí mismo en el sistema familiar. Barcelona, España: Ediciones Paidós.

Bowen, M. (1978). Family therapy in clinical practice. Nueva York, United States: Jason Aronson, Inc.

Bronfrenbrenner, U. (1987a,b,c). La ecología del desarrollo humano: Experimentos en entornos naturales y diseñados. ISBN: 84-4931086-5. Barcelona, España: Ediciones Paidós Ibérica, S.A.

Estrada, C., Herrero, J. \& Rodríguez, F. (2012). La red de apoyo en mujeres víctimas de violencia contra la pareja en el estado de Jalisco (México). Universitas Psychologica, 11(2), 523-534, e-ISSN: 16579267. Recuperado de:

https://www.redalyc.org/articulo.oa?id $=64723241014$

Fernández, R. (2005). Redes sociales, apoyo social y salud. Periferia, 3(2), 1-16, e-ISSN: 1885-8996. Recuperado de:

https://doi.org/10.5565/rev/periferia.149

Gracia, E. (1997). El apoyo social en la intervención comunitaria. 1ra edición, ISBN: 978-84-493-0352-4. Barcelona, España: Ediciones Paidós Ibérica, S.A. 
Hartman, A. (1979). Finding Families: An Ecological Approach to Family Assessment in Adoption. ISBN: 9780803912168. Nueva York, United States: Sage Publications.

INEC (2019). Encuesta nacional sobre relaciones familiares y violencia de género contra las mujeres (ENVIGMU). Ecuador: Instituto Nacional de Estadística y Censos.

La Moneda, A. (2010). Vivir, sobrevivir: el genograma, un mapa familiar. ISBN: 978-6078002023. México: Grupo Cudec.

Laso, E. (2019). Cuestionario de apoyo en pareja y estereotipos (Ecuador). Ecuador: Universidad Internacional SEK (UISEK); Universidad de Guadalajara (UdeG).

Lin, N., Dean, A., \& Ensel, W. (1986). Social Support, Life Events, and Depression. 1st Edition, ISBN: 9781483276311 . Florida, United States: Academic Press, Inc.

McGoldrick, M., \& Gerson, R. (2000a,b). Genogramas en la evaluación familiar. Tercera reimpresión, ISBN: 84-7432-493-9. Barcelona, España: Editorial Gedisa, S.A.

Minuchin, S., \& Fishman, H. (2004). Técnicas de terapia familiar. 1ra edición, ISBN: 930-12-4699-X. Argentina: Editorial Paidós SAICF.

Navarro, A., \& Rodríguez, R. (2010). Redes de apoyo en el anciano por medio del genograma y el ecomapa. Atención Familiar, 17(1), 19-21, e-ISSN: 1405-8871. Recuperado de:

http://dx.doi.org/10.22201/facmed.14058871p.2010.1.16345

OMS (2002). Informe mundial sobre la violencia y la salud: resumen. ISBN: 92-75-32422-0. Washington, D.C., Estados Unidos: Organización Mundial de la Salud.

ONU (1979). Convención sobre Eliminación de Toda Discriminación contra la Mujer (CETFDCM). Nueva York, Estados Unidos: ACNUDH.

Pérez, E. (2016). Violencia intrafamiliar hacia la mujer: Análisis 
transgeneracional desde una perspectiva sistémica en el caso de mujeres dominicanas. Tesis doctoral. España: Universidad del País Vasco. Recuperado de: http://hdl.handle.net/10810/25772

Rodrigues, R., \& Cantera, L. (2017). Violencia en la pareja: el rol de la red social. Arquivos Brasileiros de Psicologia, 69(1), 90-106, e-ISSN: 18095267. Recuperado de:

http://pepsic.bvsalud.org/scielo.php?script=sci arttext\&pid=S1809$\underline{52672017000100008}$

Satir, V. (1986). Psicoterapia familiar conjunta. 2da edición, ISBN: 968-435105-4. México, D.F.: Ediciones científicas La Prensa Medica Mexicana, S.A. de C.V.

Sluzki, C. (1996). La red social: Frontera de la práctica sistémica. Primera edición, ISBN: 84-7432-589-7. Barcelona, España: Editorial Gedisa, S.A. 
Nacida en Quito, Ecuador, el 5 de Diciembre del año

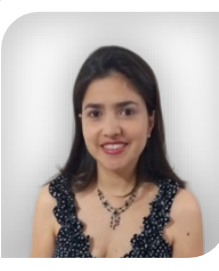
1985. Magíster en Psicología con mención en Psicoterapia por la Universidad Internacional SEK (UISEK); Diplomado en Salud Integral del Adolescente por la Universidad de Chile (UCh); Psicóloga Clínica por la Pontificia Universidad Católica del Ecuador (PUCE); con 7 años de experiencia en Salud Pública en atención primaria y hospitalaria de tercer nivel; Psicoterapeuta externa de estudiantes de la Facultad de Psicología de la Pontificia Universidad Católica del Ecuador; Experiencia en acompañamiento psicológico en el área de discapacidades en diferentes grupos etarios; Consultoría a nivel nacional en Violencia Sexual Basada en Género y Niñez en la Organización Hebrea para emigrantes (HIAS). 


\section{Elka Jacqueline Vargas Estupiñán}

e-mail: elka.vargas@uisek.edu.ec

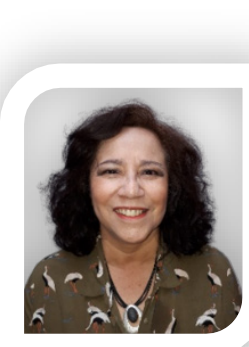

Nacida en Quito, Ecuador, el 3 de marzo del año 1963.

Doctora en Psicología educativa; Magíster en Docencia universitaria por la Universidad Tecnológica Indoamérica (UTI); y en Terapia Familiar Sistémica por la Universidad Politécnica Salesiana (UPS); Docente de la Maestría en Psicología con mención en Psicoterapia de la Universidad Internacional SEK (UISEK).

El contenido de este manuscrito se difunde bajo una Licencia de Creative Commons ReconocimientoNoComercial-Compartirlgual 4.0 Internacional 